\title{
La fabrication du soignant au travail
}

Introduction

The making of healthcarers at work. Introduction

Florent Schepens et Emmanuelle Zolesio

\section{CpenEdition}

Journals

Édition électronique

URL : https://journals.openedition.org/travailemploi/6541

DOI : 10.4000/travailemploi.6541

ISSN : 1775-416X

Éditeur

DARES - Ministère du Travail

Édition imprimée

Date de publication : 1 janvier 2015

Pagination : 5-7

ISSN : 0224-4365

\section{Référence électronique}

Florent Schepens et Emmanuelle Zolesio, «La fabrication du soignant au travail », Travail et Emploi [En ligne], 141 | janvier-mars 2015, mis en ligne le 01 janvier 2015, consulté le 04 juillet 2022. URL : http:// journals.openedition.org/travailemploi/6541; DOI : https://doi.org/10.4000/travailemploi.6541 


\title{
La fabrication du soignant au travail Introduction
}

\author{
Florent Schepens ${ }^{(*)}$ Emmanuelle Zolesio ${ }^{(* *)}$
}

Dans le contexte contemporain de l'hyperspécialisation de la médecine, comment sont formés les soignant-e-s ? Comment appréhender le travail médical comme activité et le travail de telle spécialité médicale par rapport à telle autre ? Comment devient-on soignant-e à travers les stages hospitaliers ? Telles sont les principales questions que se propose de revisiter ce dossier. Son apport principal est de donner à voir, dans un univers professionnel particulier, comment la formation s'effectue en situation de travail. Il s'agit aussi de souligner que cette formation/fabrication des soignant-e-s va bien au-delà des savoirs strictement professionnels, jouant de façon décisive dans les mécanismes de sélection avant que ne débute l'activité proprement dite, et qu'elle ne se poursuive aussi dans la suite de la carrière. Les observations établies en comparant l'acquisition du métier dans différentes spécialités et activités fournissent des éléments également utiles pour s'interroger, d'un point de vue plus théorique, sur l'objet du travail, ici médical et soignant.

Nombre d'auteurs du présent dossier poursuivent ainsi implicitement l'analyse de la socialisation professionnelle telle qu'elle a été proposée par la seconde École de Chicago. Référence incontournable, aujourd'hui encore, en sociologie de la santé comme en sociologie des professions, l'ouvrage fondateur d'Howard Becker et de ses coauteurs, Boys in White ${ }^{(1)}$ ouvrait un chantier de recherche. Les auteurs s'attachaient à découvrir les «perspectives» communes des étudiant-e-s de médecine, c'est-à-dire l'ensemble des manières de faire et de penser qui leur permettaient de faire face aux situations délicates rencontrées dans le cours de leurs études et de leur apprentissage professionnel. Leur approche abordait notamment la question du «niveau» et de la «direction» de l'effort scolaire étudiant et focalisait l'attention sur ce qui faisait la culture commune des médecins. Becker et al. soulignaient ainsi, entre autres, l'importance de l'apprentissage de la responsabilité médicale et le rôle de l'expérience pratique dans la formation clinique. Ne présentant pas leur ouvrage comme une recherche close, les auteurs invitaient à poursuivre l'analyse pour voir comment se réalisait la différenciation des spécialités médicales et, in situ, de ces cultures professionnelles distinctes vers lesquelles les candidats se sont orientés. Ce dossier s'inscrit dans la filiation de ce programme de recherche, en tentant de l'articuler avec d'autres travaux français sur la formation médicale.

Depuis Boys in White en effet, plusieurs enquêtes sur les études médicales en France se sont attachées à d'autres aspects que les «perspectives» des étudiants de médecine. Outre les propriétés sociales et le choix d'orientation des étudiant-e-s de médecine ${ }^{(2)}$, les modalités du travail universitaire académique ${ }^{(3)}$ et les pratiques de «coulisses ${ }^{(4)}$ » des études médicales ont déjà fait l'objet d'analyses sociologiques. Mais les travaux sur la formation des jeunes internes, notamment la formation clinique au cours des stages hospitaliers,

(*) Université de Bourgogne, Centre Georges-Chevrier; florent.schepens@u-bourgogne.fr

(**) Université Blaise-Pascal, membre du laboratoire ACTé (Clermont-Ferrand) et membre associé au Centre Max-Weber (Lyon) ; emmanuelle.zolesio@ens-lyon.fr

(1) Becker H. S., Geer B., Hughes E. C., Strauss A. L. (1961), Boys in white. Student culture in medical school, Chicago, University of Chicago Press.

(2) Hardy-Dubernet A.-C. (2000), «Faire (quelle?) médecine... À propos de la loi de 1982 sur la réforme du troisième cycle des études médicales», in Cresson G., Schweyer F.-X. (dir.), Professions et institutions de santé face à l'organisation du travail. Aspects sociologiques, Rennes, Éd. de l'École nationale de santé publique, pp. 87-96; Hardy-Dubernet A.-C. (2003), «L'internat de médecine ou la formation par la concurrence», in Cresson G., Drulhe M., Schweyer F.-X. (dir.), Coopérations, conflits et concurrence dans le système de santé, Rennes, Éd. de l'École nationale de santé publique, pp. 75-87; Hardy-Dubernet A.-C., Faure Y. (2006), «Le choix d'une vie... Étude sociologique des choix des étudiants de médecine à l'issue de l'examen classant national en 2005», Document de travail, Série Études, $\mathrm{n}^{\circ}$ 66, Drees.

(3) Millet M. (2003), Les étudiants et le travail universitaire. Étude sociologique, Lyon, Presses universitaires de Lyon.

(4) Pour reprendre l'expression que Geneviève Pruvost (2007, Profession, policier : sexe, féminin, Paris, Éd. de la Maison des sciences de l'homme) emprunte à Erving Goffman (1973), désignant par là les «lieu[x] où l'on a toute latitude de contredire sciemment l'impression produite par la représentation [...]. C'est là que l'acteur peut se détendre, qu'il peut abandonner sa façade, cesser de réciter un rôle» en ayant «l'assurance qu'aucun membre du public ne fera intrusion» (Goffman R. [1973], La mise en scène de la vie quotidienne. 1, La présentation de soi, Paris, Éd. de Minuit, p. 111). Emmanuelle Godeau fait une analyse de ces pratiques des étudiants de médecine, qu'elle analyse comme un «folklore» assurant la cohésion du corps médical : Godeau E. (2007), L' "esprit de corps ». Sexe et mort dans la formation des internes en médecine, Paris, Éd. de la Maison des sciences de l'homme. 
ont été relativement peu nombreux ${ }^{(5)}$. La plupart du temps, la formation des étudiant-e-s de médecine est considérée dans la globalité, à savoir dans le tronc commun des deux premiers cycles des études médicales uniquement ${ }^{(6)}$. En n'abordant quasiment jamais la spécificité du troisième cycle des études médicales, on court ainsi le risque d'homogénéiser les processus de formation. Or l'hyperspécialisation contemporaine de la médecine et l'organisation du troisième cycle des études médicales ont pour effet de différencier les soignant-e-s, par un double processus de sélection et de formation. Ce double processus, lié à «la formation de l'objet du travail médical ${ }^{(7)}$ », entraîne l'impossible confusion entre, par exemple, un médecin psychiatre, un médecin généraliste ou un neurochirurgien.

La différenciation des médecins est surtout appréhendée dans la littérature scientifique autour de la question des choix d'orientation vers les différentes spécialités en fonction des propriétés sociales et du sexe des étudiant-e-s. Les effets des réformes récentes des études de médecine, depuis le remplacement du concours de l'internat par les épreuves classantes nationales (ECN) en 2004, ont largement été étudiés par Anne-Chantal Hardy-Dubernet et font l'objet d'études statistiques renouvelées ${ }^{(8)}$. Si on connaît ainsi très bien le socle, commun à tous les étudiant-e-s de médecine, des six premières années du cursus médical et la manière dont s'effectuent les choix des étudiant-e-s au moment du second concours de leurs études (voir l'encadré 1 de l'article d'A.-C. Hardy dans ce numéro), ce dossier s'attache plus spécifiquement à la phase de différenciation des futur-e-s soignant-e-s, à l'issue de ce concours et à la professionnalisation des impétrant-e-s. Comment les étudiant-e-s en médecine, mais aussi les autres soignant-e-s sont-ils/elles formé-e-s et apprennent leur métier ? Comment sont-ils/elles socialisé-e-s à des manières de voir, dire et penser différentes de celles des autres spécialités/disciplines, au-delà du concours ?

Au-delà de la formation théorique, la fabrication du soignant, quel qu'il soit, nécessite la pratique de l'activité, à travers des stages effectués par les étudiant-e-s ou à travers une formation in situ du professionnel dans son nouveau service. Les stages sont alors d'une grande importance pour celui qui veut apprendre à soigner et si une place non négligeable est réservée à la formation "théorique» des soignant-e-s, cette dernière ne semble pas suffire pour fabriquer un-e professionnel-le. Dès lors, se pose la question de savoir ce qui est transmis à l'impétrant-e par les professionnels au cours de l'activité. Il s'agit avant tout de savoirs pratiques, de techniques et de gestes professionnels, évidemment, ce que tous les textes de ce dossier montrent, mais pas uniquement. Ainsi, si l'aide-soignant-e comme le médecin doivent travailler concrètement pour acquérir les compétences nécessaires à leur profession, l'apprentissage professionnel ne se limite pas seulement à cette dimension. Le dossier démontre notamment que les modalités de ces apprentissages sont légitimées en fonction des styles d'exercice et des visions du monde des soignant-e-s; à travers eux, ce sont aussi des dispositions qui sont transmises. Par exemple, si les chirurgiens justifient bien souvent la violence de l'apprentissage «par claques » de l'internat par la nécessité de former les internes à la violence de l'activité, les soignant-e-s de soins palliatifs insisteront sur la nécessité d'apprendre à côtoyer la mort sans violence et proposeront un apprentissage «empathique». Ainsi, les modes d'apprentissage ne sont pas identiques en fonction du segment investi ${ }^{(9)}$ et contribuent à façonner et différencier les soignant-e-s, et d'ailleurs à les sélectionner également, puisque l'accueil et les premières interactions dans le cadre des stages pratiques jouent un rôle essentiel sur l'envie de faire telle ou telle spécialité.

Il est aussi indispensable de connaître le vécu de ces situations d'apprentissage professionnel par les étudiant-e-s pour savoir comment ils et elles s'orientent vers une spécialité et deviennent un type de soignant en particulier. Les soignant-e-s, en plus des savoirs acquis dans le cadre de leur formation, sont amené-e-s également, à travers les interactions professionnelles, à apprendre leur place et leur rôle, à acquérir un certain ethos pour s'intégrer dans le collectif mais aussi à mobiliser différentes ressources sociales et professionnelles liées à leur pratique antérieure. D'où le fait que certaines spécialités apparaissent comme rédhibitoires pour certain-e-s étudiant-e-s n'étant pas en affinité avec l'ethos professionnel affiché, comme le montre la contribution de Régine Bercot pour les étudiantes qui s'écartent de la chirurgie. Avoir un diplôme et un bon rang de classement ne suffit donc pas. De même, un-e infirmier-e peut ne pas se sentir à sa place dans un service

(5) Bloy G. (2014), «La transmission des savoirs professionnels en médecine générale : le cas du stage chez le praticien», Revue française des affaires sociales, ${ }^{\circ}$ 1, pp. 103-125; Paillet A. (2007), Sauver la vie, donner la mort, Paris, La Dispute; Zolesio E. (2012), Chirurgiens au féminin? Des femmes dans un métier d'hommes, Rennes, Presses universitaires de Rennes.

(6) Saint-Marc D. (2012), La formation des médecins : sociologie des études médicales, Paris, L'Harmattan.

(7) Hardy A.-C. (2013), Travailler à guérir. Sociologie de l'objet du travail médical, Rennes, Presses de l'École des hautes études en santé publique, p. 159 et suivantes.

(8) Billaut A. (2005), "Les affectations en troisième cycle des études médicales en 2004 suite aux épreuves classantes nationales $(\mathrm{ECN}) »$, Études et résultats, no 429, Drees; Vanderschelden M. (2007), «Les affectations des étudiants en médecine à l'issue des épreuves classantes nationales en $2006 »$, Études et résultats, no 571, Drees; Fauvet L., Romain O. (collab.), Buisine S. (collab.), Laurent P. (collab.) (2012), «Les affectations des étudiants en médecine à l'issue des épreuves classantes nationales en 2011 », Études et résultats, $\mathrm{n}^{\circ} 802$, Drees, Centre national de gestion (CNG).

(9) Il peut s'agir de spécialités reconnues comme telles, ou non, comme par exemple les soins palliatifs.

- 6 - Travail et Emploi $n^{\circ} 141$ 
pédiatrique et préférer travailler en oncologie. Faire de la réanimation ou du soin palliatif ne semble pas motiver les mêmes médecins, comme le montrent Michel Castra et Florent Schepens.

Définissant «l'objet du travail» comme «ce qui donne du sens à une activité, compris à la fois comme sa direction et sa signification» (page 9), la contribution d'Anne-Chantal Hardy montre, à partir de l'étude de deux spécialités médicales (la médecine générale et la gynécologie obstétrique) deux formes d'évolutions différentes de ces objets du travail médical, fortement articulées à leur formation. Elle met également en évidence que cet objet du travail médical évolue à travers la pratique et reste dynamique aussi longtemps que le professionnel est en activité. Dès lors, comme le soulignent Michel Castra et Florent Schepens, c'est en acte qu'on devient un-e professionnel-le. Soignant-e-s, les individus le sont tous avant d'intégrer leur service; cependant, ils doivent devenir, à travers la pratique, soignant-e-s d'un service particulier, de chirurgie, de soins palliatifs, d'obstétrique, etc. Au gré de leur carrière, en fonction des services dans lesquels ils exercent, l'objet de leur travail va évoluer et la pratique professionnelle se modifier. Les trajectoires professionnelles et la pluralité des acteurs en jeu dans la formation des professionnel-le-s méritent donc également d'être interrogées et d'être prises en compte dans la durée, car les apprentissages professionnels ne se font pas qu'entre pairs pendant les études médicales, et ne s'arrêtent pas une fois les études achevées. C'était là en effet un autre enjeu de ce dossier que de considérer les soignant-e-s dans leur ensemble, en comparant les professions médicales légitimes et dotées en capitaux de toutes sortes (économique, culturel, social et symbolique) avec le «petit personnel » soignant, dans la lignée des travaux interactionnistes. Formés ensemble dans un service hospitalier, les médecins, les infirmier-e-s et les aides-soignant-e-s apprennent, dans les interactions sur le terrain, à prendre conscience de leurs places et rôles respectifs. Mais l'analyse des situations de travail et des interactions professionnelles fait aussi voir que le «petit personnel» forme en partie les futurs médecins ${ }^{(10)}$. Emmanuelle Zolesio en apporte une nouvelle preuve en montrant dans son texte que les infirmier-e-s contribuent à socialiser les chirurgiens et à infléchir parfois leurs pratiques professionnelles.

Les contributions des différents articles de ce numéro mettent aussi en évidence que cette fabrique des soignant-e-s doit être appréhendée dans la perspective dynamique de recomposition des spécialités et qu'elle participe de la formation de profils différenciés, au sein d'une spécialité, selon la génération des praticien-ne-s. On s'aperçoit par exemple que le plus ou moins grand balisage institutionnel des segments professionnels - constitués ou non comme des spécialités dans le cursus médical - comme l'évolution des configurations en termes de rapports sociaux de sexe au sein d'une spécialité contribuent de fait à la plus ou moins grande homogénéité des registres d'action des soignant-e-s. Ainsi, comme le montrent Michel Castra et Florent Schepens, les médecins qui exercent en soins palliatifs ne forment pas un sous-ensemble professionnel aux caractéristiques sociales homogènes, et l'absence de recrutement standardisé et de filière de formation organisée dans un cadre universitaire (mais aussi l'absence ou la faiblesse du registre cognitif technico-scientifique) favorisent au contraire la diversité, y compris dans les registres d'action des soignant-e-s. De même, si, dans les spécialités chirurgicales encore peu féminisées comme la chirurgie digestive, l'ethos viril semble encore s'imposer comme la norme dominante, marginalisant encore les praticiennes aux profils atypiques (Zolesio), il n'en va pas de même dans d'autres spécialités médico-chirurgicales : là où le processus de féminisation est déjà bien amorcé, c'est la pluralité d'ethos professionnels alternatifs qui semble favorisée (Bercot).

Si ce numéro de Travail et emploi porte sur les professions du soin, il nous semble que les résultats autour de l'apprentissage, de la formation in situ, du recrutement, de la socialisation professionnelle, des rapports sociaux de sexe, etc. permettent d'interroger d'autres domaines professionnels. La comparaison d'univers différents est bien souvent heuristique car elle permet de faire saillir les différences et de rendre visibles les processus analogues. «Étudier les métiers en les comparant permet de mieux comprendre le travail des médecins en étudiant celui des plombiers, et celui des prostituées en étudiant celui des psychiatres. Il ne s'agit pas de suggérer que le degré de similitude entre ces couples de métiers est plus élevé que ne permettent de l'estimer les lois du hasard, mais de préciser simplement que le chercheur part de l'hypothèse que tous les types de travail figurent sur la même liste, quel que soit leur classement sur les échelles de prestige ou de valeur morale. Pour les étudier il faut toutefois trouver un cadre de référence applicable à tous les cas, indépendamment de ces classements. C'est pourquoi nous recherchons des thèmes communs aux diverses formes de travail humain ${ }^{(11) . » ~}$

(10) Vega A. (2000), Une ethnologue à l’hôpital. L'ambiguïté du quotidien infirmier, Paris, Éd. des Archives contemporaines.

(11) Hughes E. C. (1996), Le regard sociologique : essais choisis, textes rassemblés et présentés par Jean-Michel Chapoulie, Paris, École des hautes études en sciences sociales, p. 87. 